\section{Nuevos retos en el campo de la educación avanzada del personal de enfermería en América Latina ${ }^{1}$}

En el transcurso de distintas experiencias, y particularmente a propósito del establecimiento de la meta de salud para todos en el año 2000, la OPS ha cobrado conciencia de la importancia de conocer las condiciones que imperan en América Latina en el campo de la enfermería, tanto en términos de las características del mercado de trabajo como de la estructura de los programas de estudios avanzados. Con tal fin ha venido efectuando a lo largo del tiempo algunos análisis críticos de dichas condiciones y de los factores económicos y políticos que inciden en ellas, procurando arrojar luz sobre las necesidades más perentorias en el campo de la investigación y dirigir hacia ellas la atención de las autoridades definidoras de políticas. En las páginas que siguen se examinan algunos estudios efectuados por la OPS, sus principales resultados y las distintas formas en que los cambios que actualmente experimenta el sector de la salud afectan a la profesión de la enfermería y al currículo de estudios avanzados.

\section{NECESIDADES DE INVESTIGACIÓN SOBRE EL MERCADO LABORAL EN EL CAMPO DE LA ENFERMERÍA}

Un estudio efectuado por la OPS en 1987 en seis países de la Región sirvió para poner de manifiesto las áreas de investigación de mayor interés y prioridad en el campo de la enfermería. El estudio reveló una escasez de conocimientos en las siguientes áreas: el número de miembros del personal de enfermería y su distribución por categorías y funciones; la división del trabajo en las distintas instituciones; las condiciones de trabajo y las características del mercado laboral; la organización técnica y administrativa de los servicios; los problemas que entraña el ejercicio de la profesión; y el impacto de los cuidados de enfermería en la atención primaria y en la salud de la población. A estas necesidades en el campo de la investigación se sumaron las citadas por un grupo de profesionales de la enfermería que fue convocado en 1990 en la Escuela de Enfermería de Ribeirão Prêto de la Universidad de São Paulo, Brasil, por las Coordinaciones de Investigación y Recursos Humanos de la OPS. Este grupo identificó la necesidad de investigar más a fondo el valor que se concede al trabajo de enfermería y los procesos mediante los cuales el personal mantiene y desarrolla sus habilidades.

Basado en un informe preliminar del Grupo de Trabajo sobre el mercado laboral en enfermería en el contexto de las reformas del sector de la salud, División de Desarrollo de Sistemas y Servicios de Salud, Programa de Desarrollo de Recursos Humanos, OPS, y en un documento de trabajo elaborado por Miotto Wright MG, Garzón Alarcón N. Estudio de los programas de especialización y maestría en enfermería en América Latina, Washington, DC, OPS, 1995. 


\section{LOS PROBLEMAS QUE ENFRENTA EL PERSONAL DE ENFERMERÍA}

El estudio de 1987 identificó, además, los principales problemas que enfrentan los profesionales de la enfermería, particularmente su papel mal definido en relación con el de otros profesionales del sector de la salud, la división ineficiente de las funciones dentro de un equipo de trabajo, su falta de participación en los distintos niveles de decisión y la distribución desigual del personal en áreas geopolíticas e institucionales. En general se llegó a la conclusión de que las condiciones de trabajo del personal de enfermería han sufrido un marcado deterioro.

Un análisis prospectivo de la educación en enfermería que se efectuó de 1986 a 1989 arrojó luz sobre algunas tendencias adicionales. Reveló que el personal de enfermería representa $55 \%$ de todo el personal de salud que presta servicios a la población, y que los trabajadores con títulos avanzados constituyen de 10 a $20 \%$ de todo el personal de enfermería. Por consiguiente, estos profesionales se ocupan más de la planificación que de la ejecución de las acciones. El número de personas en el campo de la enfermería no cubre las necesidades de la población y en muchas instituciones sus cargos han estado congelados por años, lo cual redunda en un gran subempleo y desempleo dentro del sector. Por último se observó una tendencia a crear más cargos de tipo auxiliar que de nivel profesional.

Según los resultados del análisis prospectivo, la estrategia de atención primaria impone al personal de enfermería nuevas responsabilidades relacionadas con el diagnóstico y tratamiento de enfermedades comunes y con el seguimiento de pacientes geriátricos o con enfermedades crónicas. Dentro de los programas de atención ambulatoria y domiciliaria se identificaron nuevas funciones que se suman a las que habitualmente desempeña el personal de enfermería en relación con el fomento de la salud y la atención del embarazo y parto.

Los enormes adelantos en las áreas de tecnología, biotecnología y comunicaciones que se han producido en décadas recientes han dado lugar a profundas transformaciones, particularmente en relación con el intercambio de bienes y servicios y la educación y salud de los pueblos. Estos adelantos han generado, simultáneamente, nuevas necesidades en el campo de la formación de recursos humanos, pues hace falta personal adepto en el manejo de los nuevos instrumentos científicos y tecnológicos y a la vez consciente de la diversidad y complejidad de los factores que entran en juego en la promoción y en el mantenimiento de la salud de la población.

Las reformas que se están llevando a cabo en el sector de la salud en América Latina han puesto de manifiesto ciertas necesidades muy urgentes en conexión con la formación del personal de enfermería. Por el momento hay poca cobertura geográfica e institucional y existe un desarrollo insuficiente de personal en áreas de importancia crítica. Las leyes que rigen al respecto se encuentran desactualizadas. Las funciones del personal de enfermería están mal definidas y hay una falta general de incentivos, especialmente para la labor en zonas rurales y en barrios marginales periurbanos.

Existen otros problemas en relación con el ordenamiento y la flexibilidad de los procesos de trabajo y la formación de los profesionales de la enfermería aún no se ha adaptado debidamente a los numerosos cambios que están sufriendo los servicios de salud. Por otra parte, las políticas generales del sector de la salud o carecen de efectividad o no se llegan a poner en práctica. Se siguen adoptando tecnologías sin examinar su verdadera utilidad. Todos estos factores han contribuido a la presencia de conflictos laborales, a la poca capacidad adquisitiva de los salarios del personal, a un empeoramiento de las condiciones de trabajo y al desprestigio de la profesión de la enfermería.

\section{MODIFICACIONES NECESARIAS EN LA FORMACIÓN DEL PERSONAL DE ENFERMERÍA}

Hay una necesidad creciente, dadas las circunstancias descritas anteriormente, de crear métodos didácticos creativos que se orienten hacia el uso simultáneo de muchos medios de comunicación, redes electrónicas y fuentes de información variadas. Es preciso reexaminar la función del profesorado, ajustar la formación del personal a las exigencias dictadas por la práctica, e incrementar la flexibilidad del currículo académico y su carácter interdisciplinario. También es necesario modificar los sistemas de acreditación y autorregulación de las instituciones y programas de estudio, y los de evaluación de alumnos y profesores. Estas modificaciones deben acompañarse de una reestructuración administrativa y de una redefinición de las tareas y responsabilidades del personal de enfermería, sin perder de vista su función esencial. Las situaciones en que se llevan a cabo las prácticas formativas deben aprovecharse para distintos fines, dando cabida a especialidades transitorias. El fomento del pensamiento crítico y la preparación de estudiantes en áreas gerenciales son aspectos adicionales que deben tenerse en cuenta en la formación del personal de enfermería. 


\section{ESTUDIO LATINOAMERICANO SOBRE LOS PROGRAMAS DE ESPECIALIZACIÓN Y MAESTRÍA EN ENFERMERÍA}

El Programa de Desarrollo de Recursos Humanos de la OPS, atento a los cambios descritos anteriormente y a sus implicaciones, ha lanzado una iniciativa pionera para estudiar la situación de los programas de posgrado en enfermería en América Latina y el Caribe. Esta iniciativa se concentra en los 32 programas de especialización y 16 de maestría que se ofrecen en siete países hispanoparlantes: Chile, Colombia, Ecuador, México, Panamá, Perú y Venezuela. Se excluyen los demás países porque no tienen cursos de posgrado o, en el caso del Brasil, porque ya abundan los estudios sobre el tema.

Se aplicaron dos grupos de cuestionarios para explorar las condiciones que dieron lugar a la creación del programa de posgrado y las características básicas del mismo en cuanto a organización, infraestructura, currículo, profesorado, alumnado y producción científica. Los programas fueron examinados, mediante un enfoque crítico y holístico, desde el punto de vista de sus componentes ideológicos, productos, orientaciones científicas y niveles de decisión micropolíticos y macropolíticos. El enfoque crítico y holístico permitió estudiar los programas de posgrado en enfermería en el contexto general de la educación superior en cada país. El primero de estos niveles se refiere a las condiciones, situación general y productos de cada programa de posgrado en enfermería; el segundo, al sistema de educación que impera en cada país, universidad y facultad.

Se partió de la base de que todo programa de posgrado en enfermería debe atender a los siguientes objetivos:

- ofrecer formación profesional avanzada;

- adiestrar profesores para la enseñanza superior;

- preparar a los estudiantes para cargos de mediano y alto nivel;

- promover el desarrollo científico;

- formar investigadores de prestigio en las ciencias básicas y aplicadas;

- fomentar ambientes propicios para la investigación;

- articular la investigación con el desarrollo de los sectores generadores de servicios.

\section{Resultados del estudio}

Según los datos recolectados en el estudio, los programas de especialización y maestría en enfermería que fueron examinados nacieron en el contexto de ideas reformistas en torno a la educación universitaria. Estas ideas se orientaban principalmente hacia la preparación de profesores para la enseñanza superior y de profesionales para el ejercicio y el desempeño de funciones gerenciales. En cambio, al desarrollo científico y tecnológico no se le otorgaba la misma importancia dentro de este marco conceptual.

El nivel macropolítico. En América Latina se tiene poca conciencia de la complejidad del sistema de posgrado en enfermería y solamente un país (Brasil, como se señaló anteriormente) ha hecho investigaciones nacionales sistematizadas sobre el tema.

El adelanto en las áreas de ciencia y tecnología depende en gran medida de los programas de posgrado. Los siete países estudiados, a diferencia de Brasil, Canadá y Estados Unidos de América, tienen sistemas de educación avanzada en enfermería que recién empiezan a formar parte de un sistema nacional organizado. El análisis crítico y holístico del nivel macropolítico reveló los siguientes obstáculos al desarrollo y a la consolidación de la educación avanzada en la Región:

- la falta de una política general para la creación, expansión y consolidación de los programas de posgrado en el campo de la salud en general y de la enfermería en particular;

- la falta de un sistema de información organizado y sistematizado, con bases de datos, sobre los programas de posgrado en cada país, universidad, o escuela de enfermería;

- la falta de criterios y procesos uniformes o coherentes para evaluar y acreditar los programas de posgrado. Tales procesos facilitarían la transferencia de alumnos y el intercambio cooperativo entre las universidades y los países;

- la dificultad para diferenciar los niveles y funciones de las distintas modalidades de posgrado (especialización, maestría o doctorado) en cuanto a su enfoque, horas académicas y número de créditos, y el perfil académico de las personas que egresan de los programas;

- la cobertura inadecuada de los programas de posgrado de especialización y maestría en términos de la formación de recursos humanos de nivel avanzado para las distintas subregiones de las Américas;

- la falta de uniformidad de los planes educativos para el personal docente de los programas de pregrado y posgrado en el campo de la salud, y especialmente de la enfermería;

- la utilización inadecuada, en el ámbito nacional, de los recursos humanos en el área de la enfermería; 
- la presencia de influencias extrarregionales en el diseño curricular de posgrado y el poco intercambio entre los países de la Región;

- la ausencia de políticas globales y de criterios para la formulación de propuestas y la definición de las características de los programas interdisciplinarios de enfermería en cada país; y

- la carencia de una política nacional y de organismos internacionales para el financiamiento de los programas de posgrado en enfermería y para fines de investigación.

El nivel micropolítico. Las condiciones en que se llevan a cabo en los países los programas de posgrado en enfermería, así como los productos de dichos programas y su situación en general, pertenecen al nivel de análisis micropolítico. La descripción crítica y holística de estos factores, que permite ubicar cada programa dentro del contexto de la comunidad académica nacional e internacional, ha revelado los siguientes obstáculos al desarrollo y a la consolidación de la educación avanzada en la Región:

- la deficiencia de los sistemas de información sobre los cupos, las matrículas y los individuos que egresan de los programas de posgrado;

- la pobreza de los sistemas de información sobre los trabajos científicos elaborados y publicados por el personal docente de los programas de posgrado, especialización y maestría;

- el alto costo de los programas de posgrado, que oscila entre US\$ 2000 y 4000 , y la escasez de becas;

- la falta de una distinción clara entre los trabajos de investigación que efectúan los alumnos de especialización y maestría y entre su currículo, sus experiencias didácticas y sus disciplinas;

- la preparación insuficiente del personal docente, que a menudo posee un título inferior al de sus alumnos, y la escasez de personal docente en general y de profesores con los títulos de maestría y doctorado en particular;

- la escasez de investigaciones de envergadura y el predominio de monografías y disertaciones elaboradas por los estudiantes;

- la incapacidad de los programas que existen actualmente para satisfacer la demanda interna de cada país y la demanda de los países vecinos;

- la presencia de un lapso excesivo entre la matrícula y la graduación de los estudiantes, sin que se sepa claramente si la demora ocurre mientras asisten a cursos, mientras preparan la tesis, o en ambas fases;

- la presencia de una razón de alumnos a investigadores de 6,3:1, cifra demasiado alta para lograr una buena orientación en la tesis;
- el número relativamente escaso de enfermeras docentes vinculadas a los programas de maestría en toda la América Latina, a excepción del Brasil;

- la poca producción científica de los programas de posgrado, en comparación con las escuelas de enfermería;

- el carácter aislado y descriptivo de las investigaciones realizadas por los alumnos y el personal docente y la relativa escasez de diseños cuantitativos o cualitativos; y

- la ignorancia del impacto que tienen los conocimientos científicos generados por los programas de posgrado en la docencia y en los servicios de enfermería.

\section{Recomendaciones}

En vista de la situación revelada por el análisis macropolítico y micropolítico de los 32 programas de especialización y 16 programas de maestría en enfermería existentes en América Latina, se han propuesto numerosas medidas correctoras, que se dividen en cinco áreas importantes. A grandes rasgos, estas incluyen la organización del sistema de educación de posgrado dentro de cada país y en toda la Región; la educación avanzada y desarrollo del personal de los programas de enfermería; la investigación y producción científica en el campo de la enfermería; la comunicación y el flujo de información entre miembros de la comunidad científica perteneciente a dicho campo; y la cooperación nacional e internacional en enfermería.

La organización de la educación de posgrado en los países y en toda la Región. La OPS y otros organismos internacionales deben ayudar a los países a definir una política global aplicable a la educación de posgrado en enfermería, tomando en cuenta las transformaciones que ha sufrido el sector de la salud, así como apoyar la movilización de recursos financieros, técnicos y políticos para cumplir con las metas y estrategias de desarrollo que se han sentado para los próximos 10 años en América Latina y el Caribe. También deben ayudar a los países a establecer sistemas de evaluación uniformes para los programas de posgrado, apoyar la creación de un grupo regional de expertos que estudien periódicamente la situación y el desarrollo de la educación en el campo de la enfermería, y fomentar la cooperación técnica y financiera entre países. Se recomienda promover, bajo la coordinación de la OPS, un taller con representantes del Banco Mundial, el Banco Interamericano de Desarrollo y la Fundación Kellogg para regular las iniciativas y los recursos destinados a mejorar la educación de pregrado y 
posgrado en enfermería en América Latina. Junto con el gobierno de cada país, los organismos internacionales formularían un plan para la expansión de los programas de posgrado, a manera de cubrir las áreas en que se generan nuevos conocimientos. Por último, se ha postulado la creación de un modelo teórico para reorganizar el posgrado y ajustarlo a las condiciones que imperan en los países latinoamericanos.

Los gobiernos de los países, junto con las universidades y escuelas de enfermería, deberán, a su vez, crear un grupo de consultores pertenecientes a los ministerios de educación a fin de definir las políticas de educación básica y avanzada en la Región. También deberán ocuparse de diseñar planes para lograr mayor coherencia entre los servicios de salud y las políticas de educación; fomentar la participación del personal de enfermería en la adopción de políticas y en el asesoramiento de los programas de ciencia y tecnología, y crear y coordinar convenios y consorcios interinstitucionales e internacionales que ayuden a fortalecer la educación de posgrado en enfermería. Será necesario, simultáneamente, establecer en los países, las universidades y escuelas de enfermería un sistema de información, aplicado a la educación de posgrado, que incluya todos los elementos propios del proceso de apertura, mantenimiento y evaluación de un programa. En general, se deberá promover el avance de la enfermería en América Latina y el Caribe mediante seminarios y talleres interdisciplinarios en el campo de la salud.

La educación avanzada del personal de enfermería. Los organismos internacionales, entre ellos la OPS, deben apoyar la formación y capacitación del personal de enfermería en los niveles de maestría y doctorado, de acuerdo con las necesidades y metas establecidas en cada país. Deberá ser su función, asimismo, estimular y apoyar técnica y financieramente la promoción del intercambio de personal docente por medio de convenios y consorcios, y la creación de fondos para becas de estudio, procurando facilitar el acceso de enfermeras procedentes de países donde no hay programas de posgrado. En las reuniones nacionales e internacionales, deberán estimular el encuentro entre grupos de expertos en educación de pregrado y posgrado con el fin de evaluar sus trabajos en el campo de la enfermería y darles continuidad. Bajo la coordinación de la OPS, los organismos internacionales también deberán llevar a cabo iniciativas conjuntas para fomentar la formación de los recursos humanos necesarios para crear nuevos programas.

Debería ser función del gobierno de cada país, con el apoyo de las universidades, establecer las necesidades prioritarias de la población y elaborar sobre esa base un plan nacional de desarrollo de recursos humanos en enfermería debidamente integrado con las iniciativas de reforma del sector de la salud. Los ministerios de educación, ciencia y tecnología deberán establecer un fondo para becas y para brindar apoyo financiero a las universidades con buenos programas de posgrado en enfermería.

La investigación y producción científica en el campo de la enfermería. Debe ser función de los organismos internacionales apoyar el asesoramiento entre los países de las Américas a fin de perfeccionar los programas de maestría en enfermería y de estimular la formación de buenos centros de investigación interdisciplinarios. Es necesario que establezcan prioridades de investigación que respondan a los planes de desarrollo de cada país y a sus políticas de salud, ciencia y tecnología, promoviendo al mismo tiempo la creación, el mantenimiento y la publicación periódica de una base de datos que contenga una lista actualizada de los estudios efectuados y de los investigadores de cada programa de posgrado en enfermería. El apoyo a la investigación, que deberá brindarse por mediación del Programa de Investigación y Desarrollo de Ciencia y Tecnología de la OPS, se verá enriquecido por la organización de encuentros nacionales y regionales sobre la contribución de la enfermería al desarrollo de la ciencia y la tecnología en la Región.

Los gobiernos y las universidades definirán las prioridades de investigación en cada país, de acuerdo con las políticas de ciencia y tecnología en vigencia, y apoyarán la creación de centros de investigación que integren la enseñanza, la práctica y las investigaciones a fin de buscar solución a los problemas que enfrentan los servicios de salud y la profesión de la enfermería. Es necesario crear cursos para el personal de enfermería sobre las distintas metodologías de la investigación, especialmente por medio de convenios y consorcios y actividades de cooperación técnica nacionales e internacionales.

La comunicación entre miembros de la comunidad científica en el campo de la enfermería. Se recomienda, entre otras medidas, incluir en el Programa de Libros de Texto de la OPS una serie titulada "Cuadernos Científicos de Enfermería" para beneficio de los programas de posgrado y la investigación en este campo en América Latina y el Caribe, y aprovechar los medios electrónicos de comunicación, tales como el CD-ROM, a fin de facilitar el intercambio de información. La producción científica de cada escuela de posgrado en enfermería se deberá aprovechar para la creación conjunta 
de todo tipo de publicaciones, entre ellas libros de texto, antologías y documentos técnicos, a manera de fortalecer los recursos bibliográficos disponibles en el campo de la enfermería.

Cada escuela de enfermería debe apoyar la creación de revistas y otras publicaciones científicas nacionales e internacionales para diseminar los diferentes cursos y trabajos de investigación, y de redes de centros y unidades docentes con bibliotecas vinculadas por sistemas de comunicaciones en continuo proceso de actualización. Es importante estimular al personal de enfermería con un doctorado a que desarrolle teorías compatibles con la realidad latinoamericana.

La cooperación nacional e internacional en el campo de la enfermería. La reciente creación de acuerdos regionales, como el Pacto Andino, facilita el desarrollo de consorcios y convenios entre las universidades con escuelas de enfermería en los distintos países. Los organismos internacionales desempeñan una función importante en este sentido y están en capacidad de utilizar las agencias oficiales de los países, como los ministerios de ciencia y tecnología, para establecer convenios con los Estados Unidos de América y Canadá y desarrollar estudios para evaluar el efecto de los acuerdos de cooperación técnica y científica en el campo de la enfermería. Se deben divulgar con más entusiasmo los documentos de la OPS sobre los organismos europeos, estadounidenses y canadienses que ofrecen apoyo económico para proyectos de investigación, trabajos de extensión y el desarrollo de otros modelos de servicios de salud.

Las universidades y gobiernos de cada país cumplirán con la función de detectar y aprovechar las oportunidades de desarrollar programas y proyectos de cooperación internacional e internacional y de identificar los organismos que ofrecen recursos técnicos y financieros para la creación de programas. La realización de talleres y seminarios para examinar los diferentes aspectos de estos progra- mas y para despertar mayor interés en ellos en el ámbito nacional e internacional serán otras funciones importantes que incumben a las autoridades y centros docentes en los países de la Región.

En resumen, el estudio de la OPS ha preparado el terreno para 1) crear un sistema de evaluación nacional y regional; 2) crear un sistema de información sistematizada sobre los programas de posgrado en cada país y en la Región; 3) diseñar un plan regional de desarrollo científico y tecnológico en el campo de la enfermería para la próxima década; y 4) ampliar y consolidar los programas de cooperación internacional entre los países de las Américas.

También se presentan elementos fundamentales para elaborar un plan de ampliación y desarrollo del posgrado en enfermería, particularmente en los programas de maestría y en los de doctorado en un futuro próximo.

\section{SYNOPSIS}

\section{New challenges in the field of advanced education for nursing personnel in Latin America}

This document examines and describes the basic characteristics of the 48 postgraduate nursing programs that exist in Latin American countries. A critical and holistic analysis model is used to identify their ideological foundations, the policies that govern them at the ministerial and institutional levels, and the conditions in which the programs operate, in an attempt to highlight areas where critical needs exist and to offer recommendations on how to satisfy them. Emphasis is placed on the capacity of international agencies, governments, and universities to improve postgraduate nursing education through the definition of priorities in light of the general reforms taking place in the health sector, the provision of support for research on nursing topics, and the coordination of cooperation initiatives at the national and international levels. 